\title{
A Random Dynamical Systems Approach to Filtering in Large-scale Networks
}

\author{
Soummya Kar, Bruno Sinopoli, and José M. F. Moura
}

\begin{abstract}
The paper studies the problem of filtering a discrete-time linear system observed by a network of sensors. The sensors share a common communication medium to the estimator and transmission is bit and power budgeted. Under the assumption of conditional Gaussianity of the signal process at the estimator (which may be ensured by observation packet acknowledgements), the conditional prediction error covariance of the optimum mean-squared error filter is shown to evolve according to a random dynamical system (RDS) on the space of non-negative definite matrices. Our RDS formalism does not depend on the particular medium access protocol (randomized) and, under a minimal distributed observability assumption, we show that the sequence of random conditional prediction error covariance matrices converges in distribution to a unique invariant distribution (independent of the initial filter state), i.e., the conditional error process is shown to be ergodic. Under broad assumptions on the medium access protocol, we show that the conditional error covariance sequence satisfies a MarkovFeller property, leading to an explicit characterization of the support of its invariant measure. The methodology adopted in this work is sufficiently general to envision this application to sample path analysis of more general hybrid or switched systems, where existing analysis is mostly moment-based.
\end{abstract}

Index Terms-Networked Control Systems, Sensor Networks, Random Dynamical Systems, Estimation Error, Weak Convergence, Sensor Schedule.

\section{INTRODUCTION}

\section{A. Background and Motivation}

Networked Control Systems (NCS) have been proposed as the paradigm to model, design and analyze control systems where the effects of computation and communication on the performance of the closed loop system cannot be neglected and need to be incorporated in the model. NCS are amenable to describe large-scale systems where components may be spatially distributed and demand the services of a communication network to exchange information. Integration of communication models traditionally renders the problem of design and analysis very complex, as the traditional mathematical machinery employed in control systems in general is not adequate to include in its framework stochastic models of communication.

A new approach capable of capturing the complexity of NCS by being able to extract fundamental limitations and relevant properties is needed. In [1] we proposed to use the Random Dynamical Systems [2] framework to characterize the problem of Kalman filtering where observations may be

The authors are with the Department of Electrical and Computer Engineering, Carnegie Mellon University, 5000 Forbes Ave, Pittsburgh, PA 15213. soummyakeandrew.cmu.edu, \{brunos, moura\}@ece.cmu.edu dropped in the communication between the sensors and the estimator, as in the case where a wireless sensor network is employed to observe the evolution of a particular dynamical system. The problem was originally analyzed in [3]. In that work the authors proved the existence of a critical packet arrival rate, below which the estimation error covariance, a stochastic variable dependent upon the realization of the packet arrival process, would have infinite mean, rendering the estimate useless.

In this case the optimal estimator would fail to track the system. The model proposed in [3] has been widely adopted and extended by several authors [4], [5], [6], [7], [8], [9], [10]. Although many present extensions to general Markov chains and account for smart sensors sending local estimates instead of observations, all the results are established with respect to mean stability, i.e., boundedness of the mean covariance. This metric is unsatisfactory in many applications, as it does not provide information about the fluctuations of the error covariance that could grow and be unusable for long time intervals. We would like to characterize the asymptotic behavior of its distribution-the goal of this paper. In [1], the authors showed how RDS can be used to fully characterize the problem, by providing conditions on the convergence in distribution of the error covariance to a stationary distribution whose support can also be explicitly characterized.

In this paper we extend the result of [1] to the case of many sensors. In particular it is not our goal to evaluate the performance of the filter with respect to a specific sensor scheduling policy or communication protocol, but to uncover the macroscopic properties of such policies and their effect on the performance of the overall systems. Under fairly general assumptions on the sensor schedule (which may be a system design objective or imposed by the random communication medium) and a minimal detectability condition, we show ergodicity of the conditional error covariance sequence and explicitly characterize the support of the resulting invariant distribution. For clarity we focus on temporally i.i.d. schedules in this paper, but note how the approach can be extended to cover more general Markovian scheduling policies. We believe that this paradigm can be extended to the verification of properties of stochastic switched or hybrid systems where nowadays the analysis tends to be moment based.

We briefly describe the organization of the rest of the paper. The problem is rigorously formulated in Section II and a description of the main results of the paper appear in Section III. Section IV presents the RDS formulation of the error covariance evolution. Several properties of the resulting 
RDS are presented in Section V, whereas, Section VI provides outlines for the proofs. Finally, Section VII concludes the paper.

\section{B. Notation}

Denote by: $\mathbb{R}$, the reals; $\mathbb{R}^{M}$, the $M$-dimensional Euclidean space; $\mathbb{T}$, the integers; $\mathbb{T}_{+}$, the non-negative integers; $\mathbb{N}$, the natural numbers; and $\mathcal{X}$, a generic space. The separable Banach space of symmetric $M \times M$ matrices is denoted by $\mathbb{S}^{M}$, equipped with the induced 2-norm. The subset $\mathbb{S}_{+}^{M}$ of positive semidefinite matrices is a closed, convex, solid, normal, minihedral cone in $\mathbb{S}^{n}$, with nonempty interior $\mathbb{S}_{++}^{M}$, the set of positive definite matrices. The cone $\mathbb{S}_{+}^{M}$ induces a partial order in $\mathbb{S}^{M}$, namely, for $X, Y \in \mathbb{S}^{M}$, we write $X \preceq Y$, if $Y-X \in \mathbb{S}_{+}^{M}$. In case $X \preceq Y$ and $X \neq Y$, we write $X \prec Y$. We also have a strong order by virtue of the non-emptiness of the interior of $\mathbb{S}_{+}^{M}$, the set of positive definite matrices $\mathbb{S}_{++}^{M}$, where we write $X \ll Y$, if $Y-X \in \mathbb{S}_{++}^{M}$.

\section{Problem Formulation}

\section{A. System Model}

We consider a discrete-time linear dynamical system being observed by a network of $N$ sensors. The signal model is given by:

$$
\mathbf{x}(t+1)=A \mathbf{x}(t)+\mathbf{w}(t)
$$

where $\mathbf{x}(t) \in \mathbb{R}^{M}$ is the signal (state) vector with initial state $\mathbf{x}(0)$ being distributed as a zero mean Gaussian vector with covariance $P_{0}$ and the system noise $\{\mathbf{w}(t)\}$ is an uncorrelated zero mean Gaussian sequence independent of $\mathbf{x}(0)$ with covariance $Q$. The observation at the $n$-th sensor $\mathbf{y}_{n}(t) \in \mathbb{R}^{m_{n}}$ at time $t$ is of the form:

$$
\mathbf{y}_{n}(t)=C_{n} \mathbf{x}(t)+\mathbf{v}_{n}(t)
$$

where $C_{n} \in \mathbb{R}^{m_{n} \times M}$ and $\left\{\mathbf{v}_{n}(t)\right\}$ is an uncorrelated zero mean Gaussian observation noise sequence with covariance $P_{n} \gg \mathbf{0}$. Also, the noise sequences at different sensors are independent of each other, the system noise process and the initial system state. Because of limited capability of the sensors, typically the dimension of $\mathbf{y}_{n}(t)$ is much smaller than that of the signal process and the observation process at each sensor is not sufficient to make the pair $\left\{\mathbf{x}(t), \mathbf{y}_{n}(t)\right\}$ observable. Thus the sensors need to collaborate and to achieve this, they share a common wireless medium to an estimator (possibly remote.) Such a medium is bit and power constrained, the channel access is opportunistic and hence at every iteration ${ }^{1}$ only a subset of the $N$ sensors are able to send their observations to the estimator. We assume there exists a randomized sensor schedule, where at each time $t$ only a subset of sensors, randomly chosen, get channel access and successfully send their observations to the estimator. We formalize this as follows:

Definition 1 (Sensor Schedule) : Let $\mathfrak{P}$ be the power set of $\{1,2, \cdots, n, \cdots, N\}$, i.e., the set of $2^{N}$ of its subsets,

\footnotetext{
${ }^{1}$ Iteration refers to the discrete time index $\mathbb{T}_{+}$.
}

including the null set. We number the elements of $\mathfrak{P}$ as $i$, where $i$ ranges from 0 to $2^{N}-1$ and w.l.o.g. assume that 0 corresponds to the null set. A sensor schedule (randomized) $\mathcal{D}$ is a probability distribution on the set $\mathfrak{P}$, such that, at time $t$, the set $\mathcal{I}(t)$ of transmitting sensors is chosen randomly according to the distribution $\mathcal{D}$ from $\mathfrak{P}$. Also, we assume that the random process $\{\mathcal{I}(t)\}_{t \geq 0}$ is an i.i.d. sequence (taking values in the set $\mathfrak{P}$ of subsets of $\{1, \cdots, N\}){ }^{2}$ i.e., the set of transmitting sensors at time $t$ is independent of the assignment at all previous times, leading to a memoryless channel.

To every schedule $\mathcal{D}$ we assign a probability vector $\lambda^{\mathcal{D}}=$ $\left[\lambda_{0}^{\mathcal{D}}, \cdots, \lambda_{2^{N}-1}^{\mathcal{D}}\right]^{T} \in[0,1]^{2^{N}}$, such that, $\sum_{i=0}^{2^{N}-1} \lambda_{i}^{\mathcal{D}}=1$ and

$$
\mathbb{P}[\mathcal{I}(t)=i \mid I(s), \quad 0 \leq s<t]=\lambda_{i}^{\mathcal{D}}, \quad \forall t \in \mathbb{T}_{+}
$$

The schedule defined above leads to temporally independent sensor assignments, but allows correlated transmissions at each time $t$ among the sensors. The model is thus fairly general, capturing the class of memoryless (temporally) transmission schemes and subsumes many existing scheduling policies in networked control systems, for example, [3]. The model is not applicable to channels with memory, however, with the framework developed in this paper we can cope with a large class of Markovian channels as explained later. For clarity of presentation, we assume the memoryless scheduling policy $\mathcal{D}$ defined above.

Suppose a particular $\mathcal{D}$ is in place. To a generic element $i$ of $\mathfrak{P}$, we assign the corresponding subset $i_{S} \subset$ $\{1,2, \cdots, N\}$ indicating the sensors contained in $i$. We denote $i_{S}$ by $i_{S}=\left\{i_{1}, \cdots, i_{\left|i_{S}\right|}\right\}$, where $\left|i_{S}\right|$ denotes the cardinality of $i_{S}$ and $i_{j}$ for $j=1, \cdots,\left|i_{S}\right|$ denotes the $j$-th sensor in the set $i_{S}$. With this notation in place, for every $i \in \mathfrak{P}$, we define

$$
\begin{gathered}
C^{i}=\left[C_{i_{1}}^{T}, C_{i_{2}}^{T}, \cdots, C_{i_{\left|i_{S}\right|} \mid}^{T}\right]^{T} \\
\mathbf{v}^{i}(t)=\left[\mathbf{v}_{i_{1}}^{T}(t), \cdots, \mathbf{v}_{i_{\left|i_{S}\right|}}^{T}(t)\right]^{T}, \quad \forall t \in \mathbb{T}_{+}
\end{gathered}
$$

and let $R^{i}$ denote the covariance of the zero mean Gaussian vector $\mathbf{v}^{i}(t)$, which we assume to be positive definite. Then, at time $t$, if some non-trivial subset of sensors reports to the estimator, i.e., if $\mathcal{I}(t) \neq \phi$, the cumulative observation $\mathbf{y}^{\mathcal{I}(t)}(t)$ arriving at the estimator is given by

$$
\mathbf{y}^{\mathcal{I}(t)}(t)=C^{\mathcal{I}(t)} \mathbf{x}(t)+\mathbf{v}^{\mathcal{I}(t)}(t)
$$

We assume that, alongside the observation, every sensor sends an acknowledgement to the estimator whenever it gets to transmit. Thus the information available to the estimator at time $t$ is given by

$$
\mathfrak{I}(t)=\left\{\mathbf{y}^{\mathcal{I}(s)}(s), \mathcal{I}(s), \quad 0 \leq s<t\right\}
$$

and the objective is to find the minimum mean squared estimator (mmse), which is the conditional mean

$$
\widehat{\mathbf{x}}(t \mid t)=\mathbb{E}[\mathbf{x}(t) \mid \mathfrak{I}(t)]
$$

\footnotetext{
${ }^{2}$ Since we identify every element of $\mathfrak{P}$ with an unique integer $i \in$ $\left\{0, \cdots, 2^{N}-1\right\}$, for all purposes, we take $\mathcal{I}(t)$ to assume values in $\left\{0, \cdots, 2^{N}-1\right\}$.
} 
Conditioned on the information sequence $\{\mathfrak{I}(t)\}$, the signal process becomes conditionally Gaussian, and the conditional mean can be recursively updated by a modified (timevarying) Kalman filter ([11].) It is sufficient to study the evolution of the one-step predictor

$$
\widehat{\mathbf{x}}(t \mid t-1)=\mathbb{E}[\mathbf{x}(t) \mid \mathfrak{I}(t-1)]
$$

and the conditional prediction error covariance

$$
P(t)=\mathbb{E}\left[(\mathbf{x}(t)-\widehat{\mathbf{x}}(t \mid t-1))(\mathbf{x}(t)-\widehat{\mathbf{x}}(t \mid t-1))^{T} \mid \mathcal{I}(t-1)\right]
$$

It can be shown that the random sequence $\{P(t)\}_{t \in \mathbb{T}_{+}}$ evolves according to a random algebraic Riccati equation (RARE) as:

$P(t+1)= \begin{cases}A P(t) A^{T}+Q & \text { if } \mathcal{I}(t)=0 \\ A P(t) A^{T}+Q-A P(t)\left(C^{\mathcal{I}(t)}\right)^{T}\left(C^{\mathcal{I}(t)} P\left(C^{\mathcal{I}(t)}\right)^{T}\right. \\ \left.+R^{\mathcal{I}(t)}\right)^{-1} C^{\mathcal{I}(t)} P(t) A^{T} & \text { otherwise }\end{cases}$

with initial condition $P(0)=P_{0}$. Under the assumption of memoryless scheduling, the sequence $\{P(t)\}$ is a Markov process whose asymptotic properties are of interest to us. To this end we define the continuous functions $f_{i}: \mathbb{S}_{+}^{M} \longmapsto \mathbb{S}_{+}^{M}$ for $i=0, \cdots, 2^{N}-1$ by

$$
\begin{gathered}
f_{0}(X)=A X A^{T}+Q \\
f_{i}(X)=A X A^{T}+Q-A X\left(C^{i}\right)^{T}\left(C^{i} X\left(C^{i}\right)^{T}+R^{i}\right)^{-1} C^{i} X A^{T}
\end{gathered}
$$

for $i=1, \cdots, 2^{N}-1$. With the above notation, the evolution of the RARE sequence $\{P(t)\}$ can be described by:

$$
P(t+1)=f_{\mathcal{I}(t)}(P(t)), \quad P(0)=P_{0}
$$

Since the sequence $\left\{f_{\mathcal{I}(t)}\right\}$ comprises of i.i.d. random maps, the process $\{P(t)\}$ is Markov. The RARE described above can be viewed as a generalization of the RARE studied in [1], where the sequence of prediction error covariance matrices involved switching between two random functions, one of which was the Lyapunov update like $f_{0}$ and the other was the Riccati update like the $f_{i}, i=1, \cdots, 2^{N}-1$ considered in eqn. (13). Hence, as will be noted later, several technical results in this paper are direct generalizations of their specific cases considered in [1].

As is the case with even deterministic single sensor systems, some form of stabilizability and detectability is needed to guarantee stability of the filtering error process. We consider the following notion of weak detectability in a networked system:

Definition 2 (Weak Detectability) : A sensor schedule $\mathcal{D}$ is called weakly detectable if there exists $i \neq 0$ in $\mathfrak{P}$ with $\lambda_{i}^{\mathcal{D}}>0$, such that the pair $\left(C^{i}, A\right)$ is detectable.

It is to be noted that the term 'weak' in the above definition not only indicates that the above is a weak condition on the sensor network, but also has implications to weak convergence (convergence in distribution) of the sequence $\{P(t)\}$ as will be justified later. Note that weak detectability defined above does not require the pair $\left(C_{n}, A\right)$ to be detectable for any sensor $n$, which may be too strong a condition, especially when the signal process is of large dimension and the individual sensors have limited observation capabilities. However, if the network is large, it is fair to assume that a subset of sensors exists, whose cumulative observations lead to a detectable system, and with some arbitrarily small but positive probability all sensors in this subset can transmit simultaneously. So far the concept of weak detectability, as stated above, has been an abstract notion and we are yet to show its effect on the long term behavior of the RARE sequence.

We also make the following assumption on the signal process:

Assumption E.1: The pair $\left(A, Q^{1 / 2}\right)$ is stabilizable, $A$ is unstable and $Q \gg 0$.

\section{B. Stability notions and scheduling protocols}

In the sequel, when considering the sequence $\{P(t)\}$, we denote the probability and expectation operators by $\mathbb{P}^{\mathcal{D}}, P_{0}[\cdot]$ and $\mathbb{E}^{\mathcal{D}, P_{0}}[\cdot]$ respectively to emphasize the dependence on the scheduling policy and the initial condition. Also, the corresponding sequence of measures induced by the RARE process on $\mathbb{S}_{+}^{M}$ is denoted by $\left\{\mu^{\mathcal{D}, P_{0}}\right\}_{t \in \mathbb{T}_{+}}$.

We study the following notions of stability of the RARE sequence:

Definition 3 (Stochastic boundedness) : A scheduling policy $\mathcal{D}$ is said to achieve stochastic boundedness for the RARE sequence, if for all initial conditions $P_{0} \in \mathbb{S}_{+}^{M}$, the sequence $\{P(t)\}$ stays stochastically bounded, i.e., for all $P_{0} \in \mathbb{S}_{+}^{M}$

$$
\lim _{K \rightarrow \infty} \sup _{t \in \mathbb{T}_{+}} \mathbb{P}^{\mathcal{D}, P_{0}}\left(\left\|P_{t}\right\|>K\right)=0
$$

Definition 4 (Moment stability) A scheduling policy $\mathcal{D}$ is said to stabilize the RARE sequence in the $k$-th moment $(1 \leq k<\infty)$ if, for all initial conditions $P_{0} \in \mathbb{S}_{+}^{M}$, the sequence $\{P(t)\}$ has bounded moments of order $k$, i.e., for all $P_{0} \in \mathbb{S}_{+}^{M}$

$$
\sup _{t \in \mathbb{T}_{+}} \mathbb{E}^{\mathcal{D}, P_{0}}\left[\left\|P_{t}\right\|^{k}\right]<\infty
$$

We often abuse terminology to call a scheduling policy stochastically bounded (s.b.) or $k$-th moment stable.

The following proposition relates the two stability notions and establishes the important connection between weak detectability and stability:

Proposition 5 Let the pair $\left(A, Q^{1 / 2}\right)$ be stabilizable and $A$ unstable. We then have the following:

i Moment stability of any order implies stochastic boundedness, i.e., if a scheduling policy $\mathcal{D}$ is moment stable of order $k$ for some $1 \leq k<\infty$, then it is stochastically bounded.

ii Weak detectability implies stochastic boundedness, i.e., a weakly detectable scheduling policy $\mathcal{D}$ is s.b.

Proof: The first part is a general property of stochastic sequences and the proof uses Chebyshev type of inequalities 
to relate probability to expectation whose details can be found in [1]. The proof of the second part is more involved and lengthy, but an immediate generalization of its specific case involving a single sensor with intermittent transmissions is studied in [1],[12] and is omitted.

Remark 6 Proposition 5 shows that stochastic boundedness is weaker than moment stability and whereas weak detectability is sufficient to ensure s.b., stronger detectability assumptions are required to conclude moment stability. This is illustrated by an example of a single sensor system as described below. Our interest in stochastic boundedness comes from the fact that it is sufficient to ensure ergodicity of the process $\{P(t)\}$, the key point of the paper. The rest of the paper concerns this important relation between stochastic boundedness (and hence weak detectability by Proposition 5) and ergodicity of the RARE sequence, and this is achieved by using tools from the theory of RDS.

\section{Example: Single sensor system with intermittent trans- mission}

For a single sensor system, a scheduling policy corresponds to assigning a probability $\bar{\gamma} \in[0,1]$ of packet transmission. This is studied in detail in [1],[12]. We assume that $\bar{\gamma}>0$ and the pair $\left(A, C_{1}\right)$ is observable, which corresponds to the notion of weak detectability in the general case. In [1],[12] it was shown that $\bar{\gamma}>0$ is a necessary and sufficient condition for stochastic boundedness if $A$ is unstable (the case of invertible $C$ was shown in [1], whereas [12] proves the general observable case), whereas mean stability of the sequence $\{P(t)\}$ requires $\bar{\gamma}$ to be greater than a critical value which increases to 1 as $A$ becomes more and more unstable (see [3] for a lower bound on this critical probability.) Moreover, in [1] it was shown that stochastic boundedness $(\bar{\gamma}>0)$ is sufficient to ensure ergodicity of the RARE process and hence we can establish that the sequence $\{P(t)\}$ converges to a unique invariant distribution even if it is not stable in the mean. In this paper, we take this further and show that a weakly detectable schedule is sufficient to ensure ergodicity of $\{P(t)\}$, even if it is not moment stable of any order $k$.

\section{Main Results: InVARIAnt Distribution}

We state the main results of the paper. Proof outlines are provided in Section VI, the details will appear elsewhere. Several key technical components of the proofs are direct generalizations of the development in [1] for the single sensor case.

The first result concerns the ergodicity of the conditional error covariance process.

Theorem 7 Let Assumption E.1 hold and the scheduling policy $\mathcal{D}$ be weakly detectable. Then there exists a unique invariant distribution $\mu^{\mathcal{D}}$ supported on the set of positive definite matrices $\mathbb{S}_{++}^{M}$, s.t. the RARE sequence $\left\{P_{t}\right\}_{t \in \mathbb{T}_{+}}$(or the sequence $\left\{\mu_{t}^{\mathcal{D}, P_{0}}\right\}_{t \in \mathbb{T}_{+}}$of measures) converges weakly to $\mu^{\mathcal{D}}$ from any initial condition $P_{0}$.
The second result explicitly determines the support of the invariant measure $\mu^{\mathcal{D}}$.

Theorem 8 Let the hypotheses of Theorem 7 hold. Define the set $\mathcal{J}(\mathcal{D}) \subset \mathfrak{P}$ as:

$\mathcal{J}(\mathcal{D})=\left\{i \in \mathfrak{P} \mid \lambda_{i}^{\mathcal{D}}>0\right.$ and the pair $\left(C^{i}, A\right)$ is detectable $\}$

For every $i \in \mathcal{J}(\mathcal{D})$ define the set $^{3}$

$$
\begin{array}{r}
\mathcal{S}_{i}=\left\{f_{l_{1}} \circ f_{l_{2}} \circ \cdots \circ f_{l_{s}}\left(P_{i}^{*}\right) \mid l_{r}\right. \\
\in\left\{0, \cdots, 2^{N}-1\right\}, \\
\left.1 \leq r \leq s, s \in \mathbb{T}_{+}\right\}
\end{array}
$$

where $P_{i}^{*}$ is the unique fixed point of the operator $f_{i}$ : $\mathbb{S}_{+}^{M} \longmapsto \mathbb{S}_{+}^{M} \cdot{ }^{4}$ We then have:

i $\mathcal{S}_{i}=\mathcal{S}_{j}$ for all $i, j \in \mathcal{J}(\mathcal{D})$.

ii The invariant measure $\mu^{\mathcal{D}}$ is supported on the closure of $\mathcal{S}_{i}$, where $i \in \mathcal{J}(\mathcal{D})$, i.e.,

$$
\operatorname{supp}\left(\mu^{\mathcal{D}}\right)=\operatorname{cl}\left(\mathcal{S}_{i}\right), \quad i \in \mathcal{J}(\mathcal{D})
$$

where cl denotes the topological closure of a set on the space $\mathbb{S}_{+}^{M}$.

Discussions: Theorem 7 establishes the ergodicity of the prediction error covariance sequence $\{P(t)\}$ under the assumption that the scheduling policy $\mathcal{D}$ is weakly detectable. The support of the invariant distribution $\mu^{\mathcal{D}}$ is explicitly characterized in Theorem 8 in terms of the network parameters. Note that, in particular, if $\mathcal{D}_{1}$ and $\mathcal{D}_{2}$ are two weakly detectable scheduling policies, such that there exists $i \in \mathfrak{P}$ with $\lambda_{i}^{\mathcal{D}_{1}}, \lambda_{i}^{\mathcal{D}_{2}}>0$ and $\left(C^{i}, A\right)$ is detectable, then

$$
\operatorname{supp}\left(\mu^{\mathcal{D}_{1}}\right)=\operatorname{supp}\left(\mu^{\mathcal{D}_{2}}\right)=\operatorname{cl}\left(\mathcal{S}_{i}\right)
$$

where $\mathcal{S}_{i}$ is defined in eqn. (18). As shown in the single sensor case ([1]), the invariant distributions $\mu^{\mathcal{D}}$ are generally not absolutely continuous w.r.t. the Lebesgue measure and the supports supp $\left(\mu^{\mathcal{D}}\right)$ are highly fractured subsets of $\mathbb{S}_{++}^{M}$.

As is noted in the proof of Theorem 7 (Subsection VI-A), the only requirement is stochastic boundedness of the sequence $\{P(t)\}$ from every initial condition. Weak detectability ensures this condition (Proposition 5) and is generally stronger than the requirement on stochastic boundedness. Thus, the conclusions of Theorem 7 may stay valid under even weaker conditions on the schedule $\mathcal{D}$, provided we are able to establish stochastic boundedness of $\{P(t)\}$ from every initial condition. However, in the absence of weak detectability, an explicit characterization of the invariant distribution, as in Theorem 8 may not be possible.

Similarly, we can extend Theorem 7 to stationary temporally Markovian schedules (i.e., the selection process $\{\mathcal{I}(t)\}$ is a stationary Markov process rather than i.i.d.). The proof and conclusions of Theorem 7 will be unchanged, as the RDS formulation of the error process requires only stationarity (see [13].) However, with a non i.i.d. selection process

\footnotetext{
${ }^{3}$ Below, in definition of $\mathcal{S}_{i}, s$ can take the value 0 , implying $P_{i}^{*} \in \mathcal{S}$.

${ }^{4}$ The stabilizability of $\left(A, Q^{1 / 2}\right)$ and the detectability of $\left(C^{i}, A\right)$ guarantee the existence of such a $P_{i}^{*}$.
} 
$\{\mathcal{I}(t)\}$, the sequence $\{P(t)\}$ no longer stays Markov and hence the assertions of Theorem 8 may not be valid.

\section{RANDOM DYNAMICAL SYSTEM FORMULATION}

In this section, we formulate the RARE process as an RDS evolving on $\mathbb{S}_{+}^{M}$. An excellent treatment of the theory of RDS can be found in [14], [13] and the concepts relevant to us are detailed in [1]. To minimize overlap with [1] and due to lack of space, we mention RDS facts, as and when required.

We start by defining a random dynamical system (RDS). We follow the notation in [14], [13].

Definition $9(R D S)$ A RDS with (one-sided) time $\mathbb{T}_{+}$and state space $\mathcal{X}$ is the pair $(\theta, \varphi)$ :

A) A metric dynamical system $\theta=\left(\Omega, \mathcal{F}, \mathbb{P},\left\{\theta_{t}, t \in \mathbb{T}\right\}\right)$ with two-sided time $\mathbb{T}$, i.e., a probability space $(\Omega, \mathcal{F}, \mathbb{P})$ with a family of transformations $\left\{\theta_{t}: \Omega \longmapsto\right.$ $\Omega\}_{t \in \mathbb{T}}$ such that

A.1) $\theta_{0}=i d_{\Omega}, \theta_{t} \circ \theta_{s}=\theta_{t+s}, \forall t, s \in \mathbb{T}$

A.2) $(t, \omega) \longmapsto \theta_{t} \omega$ is measurable.

A.3) $\theta_{t} \mathbb{P}=\mathbb{P} \forall t \in \mathbb{T}$, i.e., $\mathbb{P}\left(\theta_{t} B\right)=\mathbb{P}(B)$ for all $B \in \mathcal{F}$ and all $t \in \mathbb{T}$.

B) A cocycle $\varphi$ over $\theta$ of continuous mappings of $\mathcal{X}$ with time $\mathbb{T}_{+}$, i.e., a measurable mapping

$$
\varphi: \mathbb{T}_{+} \times \Omega \times \mathcal{X} \rightarrow \mathcal{X},(t, \omega, X) \longmapsto \varphi(t, \omega, X)
$$

B.1) The mapping $X \longmapsto \varphi(t, \omega, X) \equiv \varphi(t, \omega) X$ is continuous in $X \forall t \in \mathbb{T}_{+}, \omega \in \Omega$.

B.2) The mappings $\varphi(t, \omega) \doteq \varphi(t, \omega, \cdot)$ satisfy the cocycle property: $\forall t, s \in \mathbb{T}_{+}, \omega \in \Omega$,

$$
\varphi(0, \omega)=i d_{\mathcal{X}}, \varphi(t+s, \omega)=\varphi\left(t, \theta_{s} \omega\right) \circ \varphi(s, \omega)
$$

In a RDS, randomness is captured by the space $(\Omega, \mathcal{F}, \mathbb{P})$. Iterates indexed by $\omega$ indicate pathwise construction. For example, if $X_{0}$ is the deterministic state at $t=0$, the state at $t \in \mathbb{T}_{+}$is

$$
X_{t}(\omega)=\varphi\left(t, \omega, X_{0}\right)
$$

The measurability assumptions guarantee that the state $X_{t}$ is a well-defined random variable. Also, the iterates are defined for non-negative (one-sided) time; however, the family of transformations $\left\{\theta_{t}\right\}$ is two-sided, which is purely for technical convenience, as will be seen later.

We now show that the sequence $\left\{P_{t}\right\}$ generated by the RARE can be modeled as the sequence of iterates (in the sense of distributional equivalence) of a suitably defined RDS.

Fix a schedule $\mathcal{D}$ and define: $\left(\widetilde{\Omega}, \widetilde{\mathcal{F}}, \widetilde{\mathbb{P}}^{\mathcal{D}}\right)$, where $\widetilde{\Omega}=\mathfrak{P}$, $\widetilde{\mathcal{F}}$ is the power set of $\mathfrak{P}$ and the discrete probability measure $\widetilde{\mathbb{P}}^{\mathcal{D}}$ is defined by

$$
\widetilde{\mathbb{P}}^{\mathcal{D}}[\{i\}]=\lambda_{i}^{\mathcal{D}}, \quad \forall i \in \mathfrak{P}
$$

Also, define the product space, $\left(\Omega, \mathcal{F}, \mathbb{P}^{\mathcal{D}}\right)$, where $\Omega=$ $\times_{t \in \mathbb{T}} \widetilde{\Omega}$ and $\mathcal{F}$ and $\mathbb{P}^{\mathcal{D}}$ are the product $\sigma$-algebra and the product measure ${ }^{5}$. From the construction, a sample point $\omega \in \Omega$ is a two-sided sequence taking values in the discrete set $\mathfrak{P}=\left\{0, \cdots, 2^{N}-1\right\}$ and, since $\mathbb{P}^{\mathcal{D}}$ is the product of $\widetilde{\mathbb{P}^{\mathcal{D}}}$, the projections are i.i.d. random variables with common distribution $\mathcal{D}$. Define the family of transformations $\left\{\theta_{t}^{R}\right\}_{t \in \mathbb{T}}$ on $\Omega$ as the family of left-shifts

$$
\theta_{t}^{R} \omega=\omega(t+\cdot), \forall t \in \mathbb{T}
$$

With this, the space $\left(\Omega, \mathcal{F}, \mathbb{P}^{\bar{\gamma}},\left\{\theta_{t}^{R}, t \in \mathbb{T}\right\}\right)$ is the canonical path space of a two-sided stationary (in fact, i.i.d.) sequence equipped with the left-shift operator; hence, (e.g., [15]) it satisfies the Assumptions A.1)-A.3) to be a metric dynamical system; in fact, it is ergodic.

Recall the functions $f_{i}(X)$ in eqns. $(12,13)$. Define the function $\tilde{f}: \Omega \times \mathbb{S}_{+}^{M} \longmapsto \mathbb{S}_{+}^{M}$ by

$$
\widetilde{f}(\omega, X)=f_{\omega(0)}(X)
$$

Since the projection map from $\omega$ to $\omega(0)$ is measurable (continuous) and $f_{i}(X)$ is jointly measurable in $i, X$, it follows that $\tilde{f}(\cdot)$ is jointly measurable in $\omega, X$. Define the function $\varphi^{R}: \mathbb{T}_{+} \times \Omega \times \mathbb{S}_{+} \longmapsto \mathbb{S}_{+}$by

$$
\begin{aligned}
\varphi^{R}(0, \omega, X) & =X \\
\varphi^{R}(1, \omega, X) & =\widetilde{f}(\omega, X) \\
\varphi^{R}(t, \omega, X) & =\widetilde{f}\left(\theta_{t-1}^{R} \omega, \varphi^{R}(n-1, \omega, X)\right)
\end{aligned}
$$

It follows from the measurability of the transformations $\left\{\theta_{t}^{R}\right\}$, the measurability of $\widetilde{f}(\cdot)$, and the fact that $\mathbb{T}_{+}$is countable that the function $\varphi^{R}(t, \omega, X)$ is jointly measurable in $t, \omega, X$. Finally, $\varphi^{R}(\cdot)$ defined above satisfies Assumption B.1) by the continuity of the $f_{i}$ s, and Assumption B.2) follows by the construction given by eqns. (27-29). Thus, the pair $\left(\theta^{R}, \varphi^{R}\right)$ is an RDS over $\mathbb{S}_{+}^{M}$. Given a deterministic initial condition $P_{0} \in \mathbb{S}_{+}^{M}$, it follows that the sequence $\left\{P_{t}\right\}_{t \in \mathbb{T}_{+}}$generated by the RARE eqn. (11) is equivalent in the sense of distribution to the sequence $\left\{\varphi^{R}\left(t, \omega, P_{0}\right)\right\}_{t \in \mathbb{T}_{+}}$ generated by the iterates of the above constructed RDS, i.e.,

$$
P_{t} \stackrel{d}{=} \varphi^{R}\left(t, \omega, P_{0}\right), \forall t \in \mathbb{T}_{+}
$$

Thus, investigating the distributional properties of $\left\{P_{t}\right\}_{t \in \mathbb{T}_{+}}$ is equivalent to analyzing the distributional properties of $\left\{\varphi^{R}\left(t, \omega, P_{0}\right)\right\}_{t \in \mathbb{T}_{+}}$, which we carry out in the rest of the paper.

In the sequel, we use the pair $(\theta, \varphi)$ to denote a generic $\operatorname{RDS}$ and $\left(\theta^{R}, \varphi^{R}\right)$ for the one constructed above for the RARE.

\section{Properties of $\left(\theta^{R}, \varphi^{R}\right)$}

\section{A. Facts about generic RDS}

We review concepts on RDS (see [14], [13] for details.) Consider a generic $\operatorname{RDS}(\theta, \varphi)$ with state space $\mathcal{X}$ as in Definition 9. Let $\mathcal{X}=V_{+}$, where $V_{+}$is a closed, convex, solid, normal, minihedral cone of a real Banach space $V$. Denote by $\preceq$ the partial order induced by $V_{+}$in $\mathcal{X}$ and

\footnotetext{
${ }^{5}$ Note the difference between the measure $\mathbb{P}^{\mathcal{D}}$ and the measures $\mathbb{P}^{\mathcal{D}}, P_{0}$ defined in Subsection II-A.
} 
$<<$ denotes the corresponding strong order. For clarity the reader may assume in the following that $V=\mathbb{S}^{M}, \mathcal{X}=$ $V_{+}=\mathbb{S}_{+}^{M}$ and $\preceq,<<$ represent the order induced by positive definiteness in the space $\mathbb{S}_{+}^{M}$. In fact, the $\operatorname{RDS}\left(\theta^{R}, \varphi^{R}\right)$ considered in this paper evolves in the cone $\mathbb{S}_{+}^{M}$ of positive semidefinite matrices, where the partial order is induced by positive definiteness.

Definition 10 (Order-Preserving $R D S$ ) : A RDS $(\theta, \varphi)$ with state space $V_{+}$is order-preserving if $\forall t \in \mathbb{T}_{+}, \omega \in$ $\Omega, X, Y \in V_{+}$,

$$
X \preceq Y \Longrightarrow \varphi(t, \omega, X) \preceq \varphi(t, \omega, Y)
$$

Definition 11 (Sublinearity) An order-preserving RDS $(\theta, \varphi)$ with state space $V_{+}$is sublinear if for every $X \in V_{+}$ and $\lambda \in(0,1)$ we have

$$
\lambda \varphi(t, \omega, X) \preceq \varphi(t, \omega, \lambda X), \forall t>0, \omega \in \Omega
$$

The RDS is strongly sublinear if in addition to eqn. (32), we have

$$
\lambda \varphi(t, \omega, X) \ll \varphi(t, \omega, \lambda X), \forall t>0, \omega \in \Omega, X \in \operatorname{int} V_{+}
$$

Definition 12 (Equilibrium) A random variable $u: \Omega \longmapsto$ $V_{+}$is called an equilibrium (fixed point, stationary solution) of the $\operatorname{RDS}(\theta, \varphi)$ if it is invariant under $\varphi$, i.e.,

$$
\varphi(t, \omega, u(\omega))=u\left(\theta_{t} \omega\right), \forall t \in \mathbb{T}_{+}, \omega \in \Omega
$$

If eqn. (34) holds $\forall \omega \in \Omega$, except on set of $\mathbb{P}$ measure zero, $u$ is an almost equilibrium.

Since the transformations $\left\{\theta_{t}\right\}$ are measure-preserving, i.e., $\theta_{t} \mathbb{P}=\mathbb{P}, \forall t$, we have

$$
u\left(\theta_{t} \omega\right) \stackrel{d}{=} u(\omega), \forall t
$$

By eqn. (34), for an almost equilibrium $u$, the iterates in the sequence $\{\varphi(t, \omega, u(\omega))\}_{t \in \mathbb{T}_{+}}$have the same distribution, which is the distribution of $u$.

Definition 13 (Orbit) For a random variable $u: \Omega \longmapsto V_{+}$, we define the forward orbit $\eta_{u}^{f}(\omega)$ emanating from $u(\omega)$ as the random set $\{\varphi(t, \omega, u(\omega))\}_{t \in \mathbb{T}_{+}}$. The forward orbit gives the sequence of iterates of the RDS starting at $u$.

Although $\eta_{u}^{f}$ is the object of interest, for technical convenience (as will be seen later), we also define the pullback orbit $\eta_{u}^{b}(\omega)$ emanating from $u$ as the random set $\left\{\varphi\left(t, \theta_{-t} \omega, u\left(\theta_{-t} \omega\right)\right)\right\}_{t \in \mathbb{T}_{+}}$.

We establish asymptotic properties for the pull-back orbit $\eta_{u}^{b}$. This is because it is more convenient and because analyzing $\eta_{u}^{b}$ leads to understanding the asymptotic distributional properties for $\eta_{u}^{f}$. In fact, the random sequences $\{\varphi(t, \omega, u(\omega))\}_{t \in \mathbb{T}_{+}}$and $\left\{\varphi\left(t, \theta_{-t} \omega, u\left(\theta_{-t} \omega\right)\right)\right\}_{t \in \mathbb{T}_{+}}$are equivalent in distribution. In other words,

$$
\varphi(t, \omega, u(\omega)) \stackrel{d}{=} \varphi\left(t, \theta_{-t} \omega, u\left(\theta_{-t} \omega\right)\right), \forall t \in \mathbb{T}_{+}
$$

This follows from $\theta_{t} \mathbb{P}=\mathbb{P}, \forall t \in \mathbb{T}$. Thus, in particular, we have the following assertion.

Lemma 14 Let the sequence $\left\{\varphi\left(t, \theta_{-t} \omega, u\left(\theta_{-t} \omega\right)\right)\right\}_{t \in \mathbb{T}_{+}}$ converge in distribution to a measure $\mu$ on $V_{+}$, where $u: \Omega \longmapsto V_{+}$is a random variable. Then the sequence $\{\varphi(t, \omega, u(\omega))\}_{t \in \mathbb{T}_{+}}$also converges in distribution to the measure $\mu$.

We now introduce some notions of boundedness of RDS, which will be used in the sequel.

Definition 15 (Boundedness) Let $a: \Omega \longmapsto V_{+}$be a random variable. The pull-back orbit $\eta_{a}^{b}(\omega)$ emanating from $a$ is bounded on $U \in \mathcal{F}$ if there exists a random variable $C$ on $U$ s.t.

$$
\left\|\varphi\left(t, \theta_{-t} \omega, a\left(\theta_{-t} \omega\right)\right)\right\| \leq C(\omega), \forall t \in \mathbb{T}_{+}, \omega \in U
$$

Definition 16 (Conditionally Compact RDS) An $\operatorname{RDS}(\theta, \varphi)$ in $V_{+}$is conditionally compact if for any $U \in \mathcal{F}$ and pullback orbit $\eta_{a}^{b}(\omega)$ that is bounded on $U$ there exists a family of compact sets $\{K(\omega)\}_{\omega \in U}$ s.t.

$$
\lim _{t \rightarrow \infty} \operatorname{dist}\left(\varphi\left(t, \theta_{-t} \omega, a\left(\theta_{-t} \omega\right)\right), K(\omega)\right)=0, \omega \in U
$$

It is to be noted that conditionally compact is a topological property of the space $V_{+}$. In particular, an RDS in a finite dimensional space $V_{+}$is conditionally compact.

We now state a limit set dichotomy result for a class of sublinear, order-preserving RDS.

Theorem 17 (Corollary 4.3.1. in [13]) Let $V$ be a separable Banach space with a normal solid cone $V_{+}$. Assume that $(\theta, \varphi)$ is a strongly sublinear conditionally compact orderpreserving RDS over an ergodic metric dynamical system $\theta$. Suppose that $\varphi(t, \omega, 0) \gg 0$ for all $t>0$ and $\omega \in \Omega$. Then precisely one of the following applies:

(a) For any $X \in V_{+}$we have

$$
\mathbb{P}\left(\lim _{t \rightarrow \infty}\left\|\varphi\left(t, \theta_{-t} \omega, X\right)\right\|=\infty\right)=1
$$

(b) There exists a unique almost equilibrium $u(\omega) \gg 0$ defined on a $\theta$-invariant $\operatorname{set}^{6} \Omega^{*} \in \mathcal{F}$ with $\mathbb{P}\left(\Omega^{*}\right)=$ 1 such that, for any random variable $v(\omega)$ possessing the property $0 \preceq v(\omega) \preceq \alpha u(\omega)$ for all $\omega \in \Omega^{*}$ and deterministic $\alpha>0$, the following holds:

$$
\lim _{t \rightarrow \infty} \varphi\left(t, \theta_{-t} \omega, v\left(\theta_{-t} \omega\right)\right)=u(\omega), \omega \in \Omega^{*}
$$

In this subsection, we establish some properties of the $\operatorname{RDS}\left(\theta^{R}, \varphi^{R}\right)$ modeling the RARE.

Lemma 18 The $\operatorname{RDS}\left(\theta^{R}, \varphi^{R}\right)$ with state space $\mathbb{S}_{+}^{M}$ is orderpreserving. In other words, $\forall t \in \mathbb{T}_{+}, \omega \in \Omega, X, Y \in \mathbb{S}_{+}^{M}$,

$$
X \preceq Y \Longrightarrow \varphi^{R}(t, \omega, X) \preceq \varphi^{R}(t, \omega, Y)
$$

Also, if $Q$ is positive definite, i.e., $Q \gg 0$, it is strongly sublinear.

\footnotetext{
${ }^{6} \mathrm{~A}$ set $A \in \mathcal{F}$ is called $\theta$-invariant if $\theta_{t} A=A$ for all $t \in \mathbb{T}$.
} 
Proof: The proof uses properties of the functions $f_{0}$ (Lyapunov) and $f_{i} \mathrm{~s}$ (Riccati) and is routine given the arguments in [1] for the single sensor case. We omit it.

\section{Theorems 7,8: Proof Outlines}

We outline the proofs of Theorems 7,8. The proof of Theorem 7 relies on the RDS formulation of the RARE, and we highlight the significant steps in Subsection VI-A, the left-out details being a generalization of the single-sensor case in [1] are omitted.

The proof of Theorem 8 rests on establishing the RARE sequence as a Markov Feller process and expressing the support of the invariant distribution as the intersection of the topological lower limits of all possible orbits. Due to space constraints, we do not provide details in this paper and rather explain intuitively how it parallels the corresponding development in [1].

\section{A. Proof of Theorem 7}

Lemma 19 Consider the $\operatorname{RDS}\left(\theta^{R}, \varphi^{R}\right)$. Let Assumption E.1 hold and the scheduling policy $\mathcal{D}$ is such that the RARE sequence $\{P(t)\}$ remains stochastically bounded for every initial condition $P_{0} \in \mathbb{S}_{+}^{M}$ (by Lemma 5 this condition is satisfied, in particular, when $\mathcal{D}$ is weakly detectable.) Then, there exists a unique almost equilibrium $u^{\mathcal{D}}(\omega) \gg 0$ defined on a $\theta^{R}$-invariant set $\Omega^{*} \in \mathcal{F}$ with $\mathbb{P}^{\mathcal{D}}\left(\Omega^{*}\right)=1$ s.t. for any random variable $v(\omega)$ possessing the property $0 \preceq v(\omega) \preceq \alpha u^{\mathcal{D}}(\omega) \forall \omega \in \Omega^{*}$ and deterministic $\alpha>0$, the following holds:

$$
\lim _{t \rightarrow \infty} \varphi^{R}\left(t, \theta_{-t}^{R} \omega, v\left(\theta_{-t}^{R} \omega\right)\right)=u^{\bar{\gamma}}(\omega), \omega \in \Omega^{*}
$$

Proof: From Lemma $18,\left(\theta^{R}, \varphi^{R}\right)$ is strongly sublinear and order-preserving. It is conditionally compact because the space $\mathbb{S}_{+}^{M}$ is finite dimensional. Also, the cone $\mathbb{S}_{+}^{M}$ satisfies the conditions required in the hypothesis of Theorem 17. From the properties of the functions $f_{i}$, we note for $t>0$

$$
\varphi^{R}(t, \omega, 0)=f_{\omega(t-1)}\left(\varphi^{R}(t-1, \omega, 0)\right) \succeq Q \gg 0
$$

Thus the hypotheses of Theorem 17 are satisfied and precisely one of the assertions a) or b) holds. We show assertion a) does not hold. Assume that a) holds on the contrary. Then, there exists $P_{0} \in \mathbb{S}_{+}^{M}$ such that

$$
\mathbb{P}^{\mathcal{D}}\left(\lim _{t \rightarrow \infty}\left\|\varphi^{R}\left(t, \theta_{-t}^{R} \omega, P_{0}\right)\right\|=\infty\right)=1
$$

Then, for every $K \in \mathbb{T}_{+}$, we have

$$
\lim _{t \rightarrow \infty}\left\|\varphi^{R}\left(t, \theta_{-t}^{R} \omega, P_{0}\right)\right\|>K, \mathbb{P}^{\bar{\gamma}} \text { a.s. }
$$

It can be shown then (see [1] for details)

$$
\lim _{t \rightarrow \infty} \mathbb{P}^{\bar{\gamma}}\left(\left\|\varphi^{R}\left(t, \theta_{-t}^{R} \omega, P_{0}\right)\right\|>K\right)=1
$$

Since the above holds for every $K \in \mathbb{T}_{+}$, we have

$$
\lim _{K \rightarrow \infty} \sup _{t \in \mathbb{T}_{+}} \mathbb{P}^{\bar{\gamma}}\left(\left\|\varphi^{R}\left(t, \theta_{-t}^{R} \omega, P_{0}\right)\right\|>K\right)=1
$$

On the other hand, the stochastic boundedness of the RARE sequence and Lemma 14 imply

$$
\begin{aligned}
& \lim _{K \rightarrow \infty} \sup _{t \in \mathbb{T}_{+}} \mathbb{P}^{\mathcal{D}}\left(\left\|\varphi^{R}\left(t, \theta_{-t}^{R} \omega, P_{0}\right)\right\|>K\right) \\
= & \lim _{K \rightarrow \infty} \sup _{t \in \mathbb{T}_{+}} \mathbb{P}^{\mathcal{D}, P_{0}}\left(\left\|P_{t}\right\|>K\right) \\
= & 0
\end{aligned}
$$

This contradicts eqn. (47) and a) does not hold. Thus b) holds, and we have the result.

Lemma 19 establishes the existence of a unique almost equilibrium $u^{\mathcal{D}}$ if the schedule $\mathcal{D}$ is weakly detectable (or more generally leads to stochastic boundedness of the RARE sequence.) From the distributional equivalence of pull-back and forward orbits, it follows that the transition semigroup generated by the RARE Markov process is uniquely ergodic, i.e., the Markov process has a unique invariant probability. However, to show that the measure induced by $u^{\mathcal{D}}$ on $\mathbb{S}_{+}^{M}$ is attracting, ${ }^{7}$ eqn. (42) must hold for all initial $v$. Lemma 19 establishes convergence for a restricted class of initial conditions $v$. We need the following result to extend it to general initial conditions, whose proof follows from properties of the Lyapunov and Riccati operators.

Lemma 20 For a schedule $\mathcal{D}$, let $u^{\mathcal{D}}$ be an almost equilibrium of the $\operatorname{RDS}\left(\theta^{R}, \varphi^{R}\right)$. Then

$$
\mathbb{P}^{\mathcal{D}}\left(\omega: u^{\mathcal{D}}(\omega) \succeq Q\right)=1
$$

Proof: [Proof of Theorem 7] We now complete the proof of Theorem 7. We only highlight the key steps, the details follow the development in [1]. Let $\mu^{\mathcal{D}}$ be the distribution of the unique almost equilibrium in Lemma 19. By Lemma 20 we have $\mu^{\mathcal{D}}\left(\mathbb{S}_{++}^{M}\right)=1$. Let $P_{0} \in \mathbb{S}_{+}^{M}$ be an arbitrary initial state. Recall $\Omega^{*}$ as the $\theta^{R}$-invariant set with $\mathbb{P}^{\bar{\gamma}}\left(\Omega^{*}\right)=1$ in Lemma 19 on which the almost equilibrium $u^{\mathcal{D}}$ is defined. By Lemma 20 , there exists $\Omega_{1} \subset \Omega^{*}$ with $\mathbb{P}^{\mathcal{D}}\left(\Omega_{1}\right)=1$, such that

$$
u^{\mathcal{D}}(\omega) \succeq Q, \omega \in \Omega_{1}
$$

Define the random variable $\tilde{X}: \Omega \longmapsto \mathbb{S}_{+}^{M}$ by

$$
\begin{cases}P_{0} & \text { if } \omega \in \Omega_{1} \\ 0 & \text { if } \omega \in \Omega_{1}^{c}\end{cases}
$$

Now choose $\alpha>0$ sufficiently large, such that, $P_{0} \preceq \alpha Q$, which is possible because $Q \gg 0$. Then it can be shown

$$
0 \preceq \widetilde{X}(\omega) \preceq \alpha u^{\bar{\gamma}}(\omega), \omega \in \Omega^{*}
$$

Then, by Lemma 19, we have

$$
\lim _{t \rightarrow \infty} \varphi^{R}\left(t, \theta_{-t}^{R} \omega, \tilde{X}\left(\theta_{-t}^{R} \omega\right)\right)=u^{\bar{\gamma}}(\omega), \omega \in \Omega^{*}
$$

which implies convergence in distribution, i.e.,

$$
\varphi^{R}\left(t, \theta_{-t}^{R} \omega, \widetilde{X}\left(\theta_{-t}^{R} \omega\right)\right) \Longrightarrow \mu^{\bar{\gamma}}
$$

as $t \rightarrow \infty$. Then by Lemma 14, the sequence

\footnotetext{
${ }^{7}$ Attracting here refers to convergence in distribution to the unique invariant measure from every initial condition.
} 
$\left\{\varphi^{R}(t, \omega, \tilde{X}(\omega))\right\}_{t \in \mathbb{T}_{+}}$also converges weakly to the unique stationary distribution $\mu^{\bar{\gamma}}$.

Now, since $\mathbb{P}^{\bar{\gamma}}\left(\Omega_{1}\right)=1$, by eqn. (51)

$$
\varphi^{R}\left(t, \omega, P_{0}\right)=\varphi^{R}(t, \omega, \tilde{X}(\omega)), \mathbb{P}^{\bar{\gamma}} \text { a.s. } t \in \mathbb{T}_{+}
$$

from which we can conclude that

$$
P_{t} \Longrightarrow \mu^{\bar{\gamma}}
$$

as $t \rightarrow \infty$.

\section{B. Proof outline for Theorem 8}

The proof is lengthy and will be treated in detail elsewhere. The general line of arguments follow the corresponding in [1] for the single sensor case. It can be shown that the Markov process $\{P(t)\}$ possesses the weak Feller property, hence the support of its attracting probability is the intersection of the topological lower limits of the forward orbits emanating from all initial conditions (see, for example, [16].) The result can then be obtained by analyzing the limit properties of the maps $f_{i}$ s. We refer the interested reader to [1], which although deals with the single sensor problem, reflects the key technical arguments necessary for the development.

\section{CONCLUSIONS}

We considered a broad class of estimation problems arising in networked control systems and established the ergodicity of the optimal estimation error process under fairly general conditions. The resulting invariant distribution is not absolutely continuous w.r.t. the Lebesgue measure in general, and we explicitly identify the support of its invariant distribution. We envision the applicability of the techniques developed in this paper to the pathwise analysis of more general hybrid or switched systems.

\section{ACKNOWLEDGEMENTS}

This work was partially supported by NSF under grants \# ECS-0225449 and \# CNS-0428404.

\section{REFERENCES}

[1] S. Kar, B. Sinopoli, and J. M. F. Moura, "Kalman filtering with intermittent observations: Weak convergence to a stationary distribution," March 2009, IEEE Transactions on Automatic Control (conditionally accepted as a regular paper). [Online]. Available: http://arxiv.org/abs/0903.2890

[2] L. Arnold, Random Dynamical Systems. Springer, 1998.

[3] B. Sinopoli, L. Schenato, M. Franceschetti, K. Poolla, M. Jordan, and S. Sastry, "Kalman filtering with intermittent observations," IEEE Transactions of Automatic Control, vol. 49, no. 9, pp. 1453-1464, 2004.

[4] X. Liu and A. Goldsmith, "Kalman filtering with partial observation losses," in Proceedings of IEEE Conference on Decision and Control, vol. 4, Bahamas, December 2004, pp. 4180-4186.

[5] V. Gupta, T. Chung, B. Hassibi, and R. M. Murray, "On a stochastic sensor selection algorithm with applications in sensor scheduling and sensor coverage," Submitted to Automatica, 2005.

[6] Y. Xu and J. Hespanha, "Estimation under controlled and uncontrolled communications in networked control systems," in IEEE Conference on Decision and Control, Sevilla, Spain, December 2005, pp. 842-847.

[7] M. Huang and S. Dey, "Stability of kalman filtering with markovian packet losses," Automatica, vol. 43, no. 4, pp. 598-607, 2007.
[8] K. Plarre and F. Bullo, "On kalman filtering for detectable systems with intermittent observations," IEEE Transactions on Automatic Control, May 2007, submitted for publication.

[9] C. Robinson and P. R. Kumar, "Sending the most recent observation is not optimal in networked control: Linear temporal coding and towards the design of a control specific transport protocol," in Proc. of IEEE CDC, Dec 2007, pp. 334-339.

[10] L. Xie and L. Xie, "Stability of a random riccati equation with markovian binary switching," in Proc. of IEEE CDC, Dec 2007.

[11] R. S. Liptser and A. N. Shiryaev, Statistics of Random Processes II. Springer-Verlag, 1974.

[12] S. Kar and J. M. F. Moura, "Kalman filtering with intermittent observations: Weak convergence and moderate deviations," September 2009 , to be submitted.

[13] I. Chueshov, Monotone Random Systems - Theory and Applications. Springer, 2002.

[14] L. Arnold and I. Chueshov, "Order-preserving random dynamical systems: Equilibria, attractors, applications," Dynamics and Stability of Systems, vol. 13, pp. 265-280, 1998.

[15] O. Kallenberg, Foundations of Modern Probability, 2nd ed. Springer Series in Statistics., 2002.

[16] R. Zaharopol, Invariant Probabilities of Markov-Feller Operators and their Supports. Birkhauser, 2005. 